\title{
The effect of vessel depth, diameter, and location on ultrasound- guided peripheral intravenous catheter longevity.
}

\author{
J Matthew Fields \\ Thomas Jefferson University \\ Anthony J Dean \\ University of Pennsylvania \\ Raleigh W Todman \\ University of Pennsylvania \\ Arthur K Au \\ Thomas Jefferson University \\ Follow this and additional works at: https://jdc.jefferson.edu/emfp \\ '-nton L Anderson \\ Univertity dhe Altirnsytikikia and Complementary Medicine Commons, and the Emergency Medicine \\ Commons \\ $\frac{\text { Let us know how access to this document benefits you }}{\text { See next page for additional authors }}$
}

\section{Recommended Citation}

Fields, J Matthew; Dean, Anthony J; Todman, Raleigh W; Au, Arthur K; Anderson, Kenton L; Ku, Bon S; Pines, Jesse M; and Panebianco, Nova L, "The effect of vessel depth, diameter, and location on ultrasound-guided peripheral intravenous catheter longevity." (2012). Department of Emergency Medicine Faculty Papers. Paper 13.

https://jdc.jefferson.edu/emfp/13

This Article is brought to you for free and open access by the Jefferson Digital Commons. The Jefferson Digital Commons is a service of Thomas Jefferson University's Center for Teaching and Learning (CTL). The Commons is a showcase for Jefferson books and journals, peer-reviewed scholarly publications, unique historical collections from the University archives, and teaching tools. The Jefferson Digital Commons allows researchers and interested readers anywhere in the world to learn about and keep up to date with Jefferson scholarship. This article has been accepted for inclusion in Department of Emergency Medicine Faculty Papers by an authorized administrator of the Jefferson Digital Commons. For more information, please contact: JeffersonDigitalCommons@jefferson.edu. 


\section{Authors}

J Matthew Fields, Anthony J Dean, Raleigh W Todman, Arthur K Au, Kenton L Anderson, Bon S Ku, Jesse $M$ Pines, and Nova L Panebianco 
The effect of vessel depth, diameter, and location on ultrasound-guided peripheral intravenous catheter longevity.

By J. Matthew Fields, MD; Anthony J. Dean, MD; Raleigh W. Todman, MD; Arthur K. Au, MD; Kenton L. Anderson, MD; Bon S. Ku, MD, MPP; Jesse M. Pines, MD, MSCE; Nova L. Panebianco, MD, MPH

As published in: The American Journal of Emergency Medicine Volume 30, Issue 7, September 2012, Pages 1134-1140 
Title: The effect of vessel depth, diameter, and location on ultrasound-guided peripheral intravenous catheter longevity.

\section{Introduction:}

\section{Background}

Patients with difficult intravenous access (DIVA) present an ongoing challenge to providing emergency care and resuscitation. Of the 116.8 million ED visits in 2007 in the United States $27 \%$ required IV catheter placement. ${ }^{1}$ In many cases, failure to obtain peripheral IV access necessitates central venous cannulation (CVC), which introduces an increased risk of immediate and delayed complications secondary to a more invasive procedure and incurs increased utilization of departmental resources. ${ }^{2}$ Extrajugular vein cannulation is an alternative approach but is obtainable in less than $50 \%$ of patients and usually necessitates the involvement of a physician. ${ }^{3}$ This approach also usually requires the patient to tolerate supine or Trendelenburg positioning, which may be difficult for many patients.

Bedside ultrasound provides an alternative method of obtaining venous access when traditional landmark methods fail. ${ }^{4,5}$ However, ultrasound-guided peripheral IVs (USGPIVs) suffer from a failure rate of up to $8 \%$ in the first hour after placement and $47 \%$ within the first 24 hours after placement. ${ }^{4,6,7}$ This is much higher than traditionally placed IVs, which have reported failure rates of only 6-32\% after three days. ${ }^{8,9}$ Dislodgement of an IV has the potential to threaten patient safety by interrupting delivery of medications and fluids and putting patients at risk of extravasation that can lead to ischemia and/or tissue necrosis.

There are limited data regarding the reasons for the higher rate of failure for IVs placed under ultrasound guidance. One possible explanation is ultrasound provides the ability to access vessels that are deeper and smaller than those visible or palpable on physical exam. In addition, USGPIVs are often placed in different locations. In one study comparing the two techniques, $74 \%$ of USGPIVs were 
placed in the basilic or brachial vein; whereas $86 \%$ of traditionally placed IVs were placed in the forearm, hand, or antecubital fossa. ${ }^{10}$ Catheters that have to traverse through more subcutaneous tissue or are in atypical locations may be more likely to kink or dislodge suggesting the hypothesis that IV durability is affected by the characteristics of the vessel being cannulated. By analyzing the outcomes of IVs placed under ultrasound guidance, the current study set out to determine the influence of vessel depth, diameter, and location on IV longevity.

\section{Methods:}

\section{Study Design}

This study utilized a previously gathered database of DIVA patients who underwent USGPIV placement in the ED. This database was gathered prospectively and included images and measurements of the target vessel's depth, diameter, and location. The original analysis of this USGPIV database examined the relation between vessel depth and diameter on success of USGPIV placement and has been reported in the literature. ${ }^{11}$ For the current study an additional retrospective chart review was performed on patients in the database to determine the outcome and survival time of the USGPIVs. The institution internal review board approved the study and written informed consent was obtained for all patients.

\section{Study Setting and Population}

Patients in this database were included in the current study if they had DIVA (defined as any patient with two failed peripheral IV attempts or a history of DIVA with the inability to visualize or palpate veins on physical exam) and underwent successful USGPIV placement by one of four study sonographers from December 2007 to May 2008. The setting was an urban tertiary care university ED with a 4-year emergency medicine (EM) residency program and an emergency ultrasound fellowship. All EM residents complete a four-week ultrasound course during the PGY-2 year during which the requirements for emergency ultrasound, as recommended by the American College of Physicians 2001 
guidelines, are met. ${ }^{12}$ The USGPIVs were placed by two PGY-2 residents, one PGY-3 resident and an ultrasound fellow all of whom had met the above ACEP guidelines as well as demonstrating successful placement of at least 10 USGPIVs.

\section{Study Protocol}

Patients in the database underwent USGPIV placement with the following protocol. A tourniquet was applied to the upper extremity, which was then scanned for a target vessel using a Sonosite Micromaxx (Sonosite Inc., Bothell, WA) with a high-frequency L38/13-6MHz linear array transducer. Upon identification of a target vessel the sonologist obtained a transverse image of the vessel with measurement of the vessel depth (distance from the skin surface to the near wall of vessel) and diameter (distance from middle of near wall to middle of far wall). The location of the vessel was documented on a diagram of the upper extremity. Sterilization of the overlying skin was standardized with chlorhexidine swab and use of a sterile sheath. Only 20 gauge $48 \mathrm{~mm}$ long (Angiocath Autoguard, BD Medical Systems, Sandy, UT) catheters were used for USGPIV placement.

The sonologist attempted to place the catheter using the single-operator technique visualizing the needle enter the vein using real-time dynamic scanning. The use of transverse and/or longitudinal plane was based on sonologist preference. Successful IV placement was defined as aspiration of $5 \mathrm{ml}$ of blood and the ability to flush the line without resistance. The depth, diameter, and location of all USGPIV attempts were recorded. In addition, background demographic, historical, and physical examination information was recorded for each patient.

The final outcome of each USGPIV was determined by a retrospective chart review. Patients were excluded if an USGPIV was unable to be established as per the above protocol or if lack of charting documentation prevented an accurate estimation of IV removal. In the study institution a dedicated IV tracking form is utilized to document the status of all peripheral IVs on a continuous basis. Hospital policy mandates thrice-daily evaluation and documentation on this form of each IV access point on every patient, a record of the time of, and reasons for, IV removal, and any associated 
complications. Two blinded independent study personnel reviewed all charts and the time and reason for IV removal was extracted from the IV tracking forms. If there was a disagreement between the two reviewers on the time of IV removal or on the reason for IV removal, a third reviewer examined the chart. Patients were excluded if there was a failure of a consensus of at least 2 of the 3 reviewers on the time or outcome of the IV. The reviewers followed the successfully placed USGPIVs for 48 hours or up to the point of IV removal, whichever came first. Failures were considered IVs that infiltrated, dislodged, stopped working, or were discontinued prematurely for any reason. IVs that were removed for routine reasons such as lack of a need for ongoing IV access or patient discharge were not counted as failures.

\section{Statistical Analysis}

Descriptive statistics were performed on patient characteristics for patients with failed $v s$. nonfailed USGPIVs. Dichotomous data are presented as percentages. Continuous variables were categorized into logical groups for analysis. Vessel depth was divided into three zones: shallow $(<0.4 \mathrm{~cm})$, intermediate $(0.4 \mathrm{~cm}-1.19 \mathrm{~cm})$, and deep $(\geq 1.2 \mathrm{~cm})$. IV diameter was divided into four groups $(<0.3 \mathrm{~cm}, 0.3-0.39 \mathrm{~cm}, 0.4-0.49 \mathrm{~cm}$, and $\geq 0.5 \mathrm{~cm})$. Vessel location was divided into proximal (brachial region) and distal (antecubital fossa, forearm, or hand veins). The Fisher exact test and $\chi^{2}$ test were used for comparisons of categorical variables, as appropriate. Follow-up began at the time and date of successful USGPIV placement and ended at the time and date of documented USGPIV failure or removal. In order to account for patient censoring (IVs that were removed for routine reasons such as patient discharge prior to the end of the 48 hour follow-up period) USGPIV longevity was analyzed using a Kaplan-Meier survival algorithm. Sample size was determined using the Log-Rank Freedman method to compare two survival functions. Based on previous data $50-60 \%$ of USGPIVs fail at 48 hours. At least 92 patients were required to detect a 15\% difference in survival for a given covariate assuming an alpha of 0.05 and a power of 0.80 .

Univariate analysis was performed on vessel characteristics (depth, diameter, and location) 
using the log-rank test of equality. Significant predictors in the univariate analysis ( $\mathrm{p}$ value of $<0.05$ ) were included in a Cox proportional hazard regression analysis. This multivariate model checked for possible interactions between variables. Proportionality of the model was checked by visual inspection of the graphed Schoenfield residuals and goodness of fit was checked using Cox-Snell residuals. Stata 11 was used for the analysis (StataCorp, College Station, TX).

\section{Results:}

There were 183 patients in the database of which 18 patients were excluded for failure to successfully establish an USGPIV and 14 patients were excluded due to lack of sufficient documentation (12) or reviewer disagreement on IV outcome (2), yielding a total of 151 patients for analysis. The mean age of the patients was 53 years (SD \pm 18 years). Ninety-six $(64 \%)$ patients were female and 115 (76.7\%) were African American. 22 USGPIVs $(15 \%)$ were placed in shallow $(<0.4 \mathrm{~cm})$ vessels, $106(70 \%)$ were placed in intermediate vessels $(0.4-1.19 \mathrm{~cm})$, and $23(15 \%)$ were placed in deep vessels ( $\geq 1.2 \mathrm{~cm})$. There were 22 USGPIVs (15\%) placed in vessels with a diameter $<3 \mathrm{~mm}, 55$ USGPIVs (36\%) placed in vessels 3-3.9mm, 46 USGPIVs (30\%) placed in vessels 4-4.9mm, and 28 USGPIVs (19\%) placed in vessels $\geq 5 \mathrm{~mm}$. There were 95 USGPIVs $(63 \%)$ placed proximally in the brachial region and $56(37 \%)$ placed distally in the antecubital fossa or forearm veins. No USGPIVs were placed in the wrist or hand.

After 48 hours, 48 USGPIVs (32\%) had failed due to IV infiltration, dislodgement, or patient discomfort. Thirty-six USGPIVs (24\%) had been removed for routine reasons, such as patient discharge or no further need for intravenous access, and the remaining 67 USGPIVs (44\%) were still in place and without incident. Of the 48 USGPIV failures 20 IVs (42\%) infiltrated, 11 (23\%) dislodged, $16(33 \%)$ were not flushing, and 1 IV (2\%) was removed due to patient discomfort. There were no significant differences in the patient characteristics of IVs that failed vs. IVs that did not fail within 48 hours (table 1).

\section{Survival Analysis}


The Kaplan-Meier survival analysis yielded an USGPIV survival probability of 0.63 (95\%CI 0.53-0.70, Figure 1) at 48 hours. Analysis of vessel characteristics using the log-rank test for equality revealed vessel depth, location, and diameter each potentially had a significant association with IV longevity. When placed in the Cox multivariate regression model and checking for interactions between variables both vessel depth and location were found to each be independently and significantly associated with USGPIV longevity, however vessel diameter was not.

\section{Vessel Depth}

USGPIV survival probability was excellent (1.00) for shallow vessels $(<0.4 \mathrm{~cm}, \mathrm{n}=22)$, moderate $(0.62,95 \%$ CI $0.51-0.71)$ for intermediate vessels $(0.41-1.19 \mathrm{~cm}, \mathrm{n}=106)$, and poor $(0.29,95 \%$ CI 0.11 51) for deep vessels $(\geq 1.2 \mathrm{~cm}, \mathrm{n}=23), \mathrm{p}<0.0001$. Shallow and deep survival curves became significantly different as early as 3 hours after placement and all three zones were significantly different from each other from 12 hours onwards (figure 2a, Table 2).

\section{Vessel Location}

USGPIV placement in the antecubital fossa or forearm was associated with improved survival probability when compared to more proximal placement in the region of the brachial or basillic veins (figure $2 b$, Table 2). The survival curves for vessel location became significantly different at 18 hours with a survival probability of 0.93 (95\%CI 0.82-0.97) for distal vessels versus 0.71 (95\%CI 0.60-0.80) for proximal vessels.

\section{Multivariate Model}

In the Cox regression analysis depth and location were each independently associated with IV longevity. For each increase of $0.2 \mathrm{~cm}$ in vessel depth the odds of an IV failing within 48 hours increases by a hazard ratio of 1.36 [1.15-1.61]. Placement of an IV in the brachial region versus the forearm or antecubital fossa increases the likelihood of failure by a hazard ratio of 2.76 [1.25-6.09]. The multivariable model satisfied criteria for proportionality and Cox-Snell residuals did not suggest a lack of goodness of fit for the model $(\mathrm{p}=0.17)$. Utilizing these hazard ratios allows us to create a 
model to predict the survival curve for a given vessel depth and location. Examples of predicted survival curves for various permutations of vessel depth and location using this model are demonstrated (figure 3).

\section{Discussion:}

The aim of this study was to determine the effect of vessel parameters on the survival of USGPIVs. While longevity of USGPIVs has been evaluated in other studies this is the first study to examine the relationship of vessel characteristics and USGPIV survival. ${ }^{7}$ The study found that vessel depth and location were both predictive of early failure of USGPIVs. The vessel characteristic that was most strongly associated with failure was depth from skin surface and this effect seemed to occur more rapidly when compared to other vessel characteristics. As early as three hours USGPIVs placed in shallow vessels $(<0.4 \mathrm{~cm})$ had significantly better survival than those placed in deep vessels $(\geq 1.2 \mathrm{~cm})$. By 12 hours over half of USGPIVs in deep vessels fail. Vessel location had a delayed but also independently significant effect on IV survival. Within 18 hours of placement, USGPIVs placed in the antecubital fossa or forearm had better survival compared with those placed in the brachial or basillic veins.

Two studies have shown that successful USGPIV placement is influenced by vessel diameter and depth. ${ }^{11,13}$ The current study shows that after successful cannulation the continued survival of the IV is also influenced by depth, and, in addition, by IV location. Vessel diameter did not affect catheter survival after successful placement. Ideally, the sonologist would be able to select the vein with the greatest likelihood of both successful placement and longevity from a number of possible choices. However, when the choice is limited, as is often the case in patients with DIVA, knowledge of these relationships can aid the sonographer in choosing the optimal vessel for success and durability. When no suitable vessels exist the sonographer can opt to select a different method of IV access (midline catheter, intraosseus line, or CVC) and not subject the patient to unnecessary IV sticks. In the case 
where an IV is needed for only a brief period, depth should be the main consideration. When using a $48 \mathrm{~mm}$ catheter, vessels $\geq 1.2 \mathrm{~cm}$ should be avoided altogether since they have a high immediate failure rate. When an IV is needed for a longer period ( $\geq 18$ hours), the sonographer should additionally consider the importance of vessel location attempting to avoid vessels in the brachial region.

To our knowledge there are no studies specifically investigating the basis of the association between deeper and proximal veins and poor IV survival; although the finding is unlikely to surprise emergency physicians familiar with the challenges of maintaining IV access in this patient population. With deeper vessels a shorter portion of catheter tip ends up in the vessel and is more easily dislodged. In addition, with a constant catheter length, deeper vessels require inserting the catheter at a steeper angle. This has several theoretical adverse consequences. First, it makes it increasingly likely that the tip of the introducer needle pierces the back wall of the vessel prior to full introduction of the plastic catheter, resulting in damage to the vessel (predisposing to both leakage and thrombosis). Second, if the back wall of the vessel is not punctured, there is an increased risk of the catheter tip getting caught up on it or traumatizing the endothelium as it is advanced. Third, the sharp angle between the subcutaneous path of the catheter and the vessel often results in kinking when it changes its direction to conform to the vessel. This severely limits flow of intravenous fluids as well as being another potential source of trauma and/or thrombosis in the vessel.

With respect to location, the subcutaneous fat of the arm is often thicker and looser than that in the antecubital fossa or forearm. ${ }^{14}$ This makes it incrementally vulnerable to failure, both due to the reasons discussed above, and also due to the instability, mobility, and mass of the surrounding loose adipose tissue. This may be exacerbated by the tendency to compress the subcutaneous tissue to facilitate cannulation. When these tissues are released and re-expand after successful placement of the USGPIV, there is distortion of the catheter track with the potential for dislodgement or kinking. The mobility of the shoulder joint, as well as the location of the basilic and brachial veins on the medial side of the arm may also render these sites at greater risk of mechanical dislodgement. The only study to 
cast light on this issue, the one by Mills et al., supports the notion that short catheter length may be a cardinal flaw in deeper vessels. ${ }^{6}$ In that study a standard length catheter was used to cannulate the vein, but this was immediately followed by placement of a $15 \mathrm{~cm}$ long catheter over a guide wire. The only catheter of 24 subsequently to fail (median duration of catheter survival 26 hours) did so after 15 minutes. This study used proximal veins exclusively, making these findings particularly striking compared to ours in which $66 \%$ of patients in this group had lost their IVs within 24 hours. Notably the findings of Dargin et al., using a $63.5 \mathrm{~mm}$ catheter, were very similar to ours. ${ }^{7}$ Further studies are needed to identify the minimal length and optimal catheter type for improved IV longevity in these deeper peripheral veins.

\section{Limitations}

Potential bias may have occurred as a result of the outcome variable (IV longevity) being obtained by retrospective chart review. The reliability of this outcome is contingent on rapid realization of IV failure and clear documentation of the event by hospital personnel. While there were some charts that lacked clear documentation of IV outcome (12/183), there was little disagreement between the two reviewers (2/183). Furthermore, in spite of the strict hospital policies regarding documentation about IV access sites, it is possible that failed catheters were documented as "removed" either because they were no longer needed, or another was easily obtained, or to avoid the appearance of an adverse event. In addition, it is in the nature of an intravenous failure that it can only be detected after it has occurred, and IV failures are rarely detected immediately. All these factors would lead to bias towards the appearance of artifactually increased longevity. Stated more simply: our study necessarily underestimated the failure rate of the USGPIVs.

Some factors that may have affected the lifespan of the IVs were not collected. These include the nature of the infusions running through the IV, how often the IV was accessed, movement of the extremity, or whether any fixation device such as an arm-board was utilized. Additionally, there was no 
standardization to the method for which the IV was secured. In our institution peripheral IVs are routinely secured by use of a Tegaderm ${ }^{\mathrm{TM}}$ and other commercially available fixation devices such as arm boards are rarely used. It is possible that USGPIV longevity could be improved by post-procedural methods, however this was not assessed in this study.

The generalizability of the study may be limited since this was a single-center study, and with a relatively small cohort of sonologists obtaining data, it is likely that their skills with USGPIV cannulation are superior to the average among emergency physicians. However the one-hour $10 \%$ early IV failure rate seen in our study is similar to failure rates documented in other studies. ${ }^{4,7}$ The early USGPIV failure depth of $1.2 \mathrm{~cm}$ found in our study was based on using a catheter length of $48 \mathrm{~mm}$. As noted above, it is likely that altering either the catheter length or the angle of approach would affect the depths at which USGPIV longevity is compromised. In addition, only one catheter gauge was used (20gauge). This variable was controlled in the database in order to assess the relationship of other variables on USGPIV success rates. Interestingly, a study by Catney et al found that 18 gauge IVs were more durable than 20 or 22 gauge IVs suggesting that larger diameter thicker catheters may be less susceptible IV kinking and failure. ${ }^{8}$ Logically, this finding would be true for USGPIVs, however this was not assessed in the current study.

A question still remains as to whether ultrasound guidance is itself responsible for the short survival of these lines or whether this is due to some other factor (e.g. relatively short catheter length, the instability of thick subcutaneous adipose tissue, or greater friability of deeper veins). Since IV access using landmark techniques (often in the basilic region) has been performed for decades it seems likely that deeper veins accessed by any method would have an equally poor prognosis. To determine whether this is actually so will require further study.

\section{Conclusion:}

Ultrasound provides a useful rescue method for establishing intravenous access in patients with 
DIVA. The current study reveals two factors that significantly affect the durability of these IVs - depth and location. Using a $48 \mathrm{~mm}$ catheter, vessels $\geq 1.2 \mathrm{~cm}$ deep have a high likelihood of USGPIV failure and should only be cannulated when other options are not available. Vessels $<0.4 \mathrm{~cm}$ deep yield the best USGPIV longevity. Forearm and antecubital sites are more enduring than those in the upper arm. Understanding of these associations will help the sonologist select the optimal vessel for successful USGPIV cannulation and longevity.

\section{References:}

1. Niska R, Bhuiya F, Xu J. National Hospital Ambulatory Medical Care Survey: 2007 emergency department summary. National health statistics reports. 2010;(26):1-31.

2. McGee DC, Gould MK. Preventing complications of central venous catheterization. The New England journal of medicine. 2003;348(12):1123-33.

3. Costantino TG, Kirtz JF, Satz WA. Ultrasound-Guided Peripheral Venous Access vs. the External Jugular Vein as the Initial Approach to the Patient with Difficult Vascular Access. The Journal of emergency medicine. 2010;39(4):462-7.

4. Keyes LE, Frazee BW, Snoey ER, Simon BC, Christy D. Ultrasound-guided brachial and basilic vein cannulation in emergency department patients with difficult intravenous access. Annals of emergency medicine. 1999;34(6):711-4.

5. Costantino TG, Parikh AK, Satz WA, Fojtik JP. Ultrasonography-guided peripheral intravenous access versus traditional approaches in patients with difficult intravenous access. Annals of emergency medicine. 2005;46(5):456-61.

6. Mills CN, Liebmann O, Stone MB, Frazee BW. Ultrasonographically guided insertion of a 15-cm catheter into the deep brachial or basilic vein in patients with difficult intravenous access. Annals of emergency medicine. 2007;50(1):68-72.

7. Dargin JM, Rebholz CM, Lowenstein RA, Mitchell PM, Feldman JA. Ultrasonography-guided peripheral intravenous catheter survival in ED patients with difficult access. The American journal of emergency medicine. 2010;28(1):1-7.

8. Catney MR, Hillis S, Wakefield B, et al. Relationship between peripheral intravenous catheter Dwell time and the development of phlebitis and infiltration. Journal of infusion nursing : the official publication of the Infusion Nurses Society. 24(5):332-41.

9. Bregenzer T, Conen D, Sakmann P, Widmer a F. Is routine replacement of peripheral intravenous catheters necessary? Archives of internal medicine. 1998;158(2):151-6.

10. Bauman M, Braude D, Crandall C. Ultrasound-guidance vs. standard technique in difficult vascular access patients by ED technicians. The American journal of emergency medicine. 2009;27(2):135-40. 11. Panebianco NL, Fredette JM, Szyld D, et al. What you see (sonographically) is what you get: vein and patient characteristics associated with successful ultrasound-guided peripheral intravenous placement in patients with difficult access. Academic emergency medicine : official journal of the Society for Academic Emergency Medicine. 2009;16(12):1298-303.

12. Anon. American College of Emergency Physicians Emergency Ultrasound Guidelines. Annals of Emergency Medicine. 2001;38(4):470-81. 
13. Witting MD, Schenkel SM, Lawner BJ, Euerle BD. Effects of vein width and depth on ultrasoundguided peripheral intravenous success rates. The Journal of emergency medicine. 2010;39(1):70-5. 14. Botte MJ, Gelberman RH. Acute compartment syndrome of the forearm. Hand clinics. 1998;14(3):391-403. 